\title{
Aprendizajes ausentes en la Competencia Digital de preadolescentes: un estudio de casos pertenecientes a contextos socioculturales desfavorables
}

\author{
Absent learnings in pre-teens' Digital Competence: \\ a case study belonging to unfavourable socio-cultural contexts
}

\author{
Fernando Fraga-Varela \\ Universidade de Santiago de Compostela. Santiago de Compostela, España \\ fernando.fraga@usc.es \\ Esther Vila-Couñago \\ Universidade de Santiago de Compostela. Santiago de Compostela, España \\ esther.vila@usc.es \\ Eulogio Pernas-Morado \\ Universidade de Santiago de Compostela. Santiago de Compostela, España \\ eulogio.pernas@usc.es
}

\begin{abstract}
Resumen
El artículo presenta un análisis de la Competencia Digital de dos preadolescentes pertenecientes a contextos socioculturales desfavorables en el último curso de Educación Primaria. Sobre la base de una metodología de estudio de caso, con múltiples técnicas e informantes, se investiga el proceso de apropiación de la Competencia Digital y el papel de los agentes educativos en el desarrollo de la misma, tomando como referencia su Red Personal de Aprendizaje (en inglés PLN, Personal Network Learning). Los resultados muestran dos contextos muy distintos: uno con numerosos dispositivos digitales que permiten el acceso a innumerables recursos y otro con disponibilidad limitada de estos artefactos. Sin embargo, ambos casos convergen en que varias dimensiones de la Competencia Digital analizadas están escasamente desarrolladas, con la influencia de los agentes educativos, especialmente los que están presentes en el hogar. Encontramos razones clave en una escuela que muestra muchas dificultades y obstáculos para compensar su desarrollo como ciudadanos del siglo XXI.
\end{abstract}

Palabras Clave: Competencia Digital, TIC, preadolescentes, computación 1 a 1, Educación Primaria

\begin{abstract}
The article presents a Digital Competence analysis of two pre-teens belonging to unfavourable socio-cultural contexts in the last course of Primary Education. Based on a case study methodology, with multiple techniques and informants, the process of appropriation of Digital Competence and the role of the educational agents in its development is inquired, taking as reference their PLN -Personal Network Learning. The results show two very distinct contexts: one with numerous digital devices that allow access to myriad resources and another with limited availability of these artifacts. However, both cases converge in that several dimensions of the Digital Competence analyzed are poorly developed, with the influence of educational agents, especially those that are present at home. We found key reason because the school have many difficulties and obstacles to compensate their development as citizens of the 21 st century.
\end{abstract}

Keywords: Digital Competence, ICT, pre-teen, 1:1 computing, Primary Education 


\section{Introducción}

La Competencia Digital (CD) se ha vuelto un requisito indispensable para vivir en una sociedad cada vez más conectada. Se hace necesario el desarrollo de habilidades, conocimientos y actitudes que nos permitan acceder a dispositivos y contenidos digitales (Cobo, 2019), teniendo la posibilidad de participar de manera reflexiva (McGrew, Breakstone, Ortega, Smith y Wineburg, 2018) en este nuevo escenario. Pero como señalan las investigaciones, el elemento clave de esta cuestión "radica principalmente en lo que el individuo puede hacer cuando está conectado, de qué manera puede obtener provecho para desarrollar nuevas competencias de manera que uno pueda discriminar y regular su uso dependiendo del contexto y las necesidades" (Gallardo-Echenique, 2019, p.1). Se están generando brechas, nuevas periferias entendidas como formas de exclusión social de aquellos que no saben moverse en un nuevo ecosistema digital (Velásquez Benavides, Rodríguez Hidalgo y Suing, 2018), lo que exige el desarrollo de una alfabetización digital crítica (Pérez-Escoda, García-Ruiz y Aguaded, 2019).

Prensky (2001) insistía en la idea de que la generación nacida en las últimas dos décadas del siglo XX, criada y socializada en similares entornos tecnológicos (Linne, 2018), posee habilidades que les permiten apropiarse de los dispositivos digitales con una mayor facilidad que las generaciones anteriores. Algunas investigaciones cuestionaban esta afirmación (Bennett, Maton y Kervin, 2008; Helsper y Eynon, 2009) señalando que la promesa de una generación capaz de entender por sí misma el funcionamiento de nuevas herramientas es falsa: se hace un uso muy superficial y en cualquier caso la CD es bastante limitada. Además, demuestran una preocupante falta de formación (Lluna y Pedreira, 2017) y, como indica Sorrentino (2018), la metáfora de los nativos digitales parece ser engañosa y conceptualmente confusa. Ante esta situación, urge avanzar en la comprensión de las formas en las que las nuevas generaciones se enfrentan a los retos de un uso competente de las posibilidades que los nuevos escenarios digitales proporcionan. La indagación en esta realidad permitirá poder brindar nuevas oportunidades para poder enfrentarnos a la brecha digital entendida como la distancia entre los sujetos en cuanto a la apropiación de las posibilidades que las tecnologías digitales y el uso de internet ofrecen en la actualidad (OECD, 2001).

La investigación que se presenta en este artículo parte de esta hipótesis, de una realidad en la que se espera que los más jóvenes sean Competentes Digitales y en la que la disposición de recursos en el hogar o su acceso les acerque a lo que se demanda de ellos en la sociedad. Se abordan de manera más amplia los entornos en los que se mueven los preadolescentes y cómo dichos entornos condicionan un bagaje en el que participan otros miembros de su Red Personal de Aprendizaje (PLN). Este concepto, propuesto por Tobin (1998), entiende el PLN como una red de personas, información y recursos que un individuo desarrolla mediante tecnologías sociales para acceder al aprendizaje informal (Oddone, Hughes y Lupton, 2019). Para Couros (2010) se entendería como "la suma de todo el capital social y las conexiones que dan lugar al desarrollo y la facilitación de un entorno de aprendizaje personal" (p. 125). Se trata de un concepto con una cierta trayectoria y que ya hace tiempo, autores como Warlick (2009) indicaban que podía tener tres tipos principales de accesos: mediante conexiones sincrónicas personales, haciendo consultas y buscando consejos en personas de su entorno; a través de conexiones semisincrónicas a través de una comunidad virtual de personas con las que interacciona; y finalmente de forma asincrónica a través de fuentes digitales de contenidos mantenidas por determinados sujetos, que son considerados como referentes en un tema determinado.

Aprendizajes ausentes en la Competencia Digital de preadolescentes: un estudio de casos pertenecientes a contextos socioculturales desfavorables. Fernando Fraga-Varela, Esther VilaCouñago y Eulogio Pernas-Morado.

Página 2 de 18 
En cualquier caso, entendemos PLN como una red informal de los sujetos a través de los que verdaderamente se facilita el acceso a información que posibilite un conocimiento autónomo.

En este contexto surge la necesidad de investigar la realidad del desarrollo de la CD en el alumnado de la etapa de Educación Obligatoria comprendida entre los 6 y los 12 años (denominada Educación Primaria en España) a través de estudios de casos. El trabajo permite descubrir situaciones en un juego de inclusión-exclusión y revelar sus múltiples ámbitos de proyección y consecuencias.

\section{La Competencia Digital: más allá de lo escolar}

La CD es una de las 7 competencias clave que se incorporan al Sistema Educativo Español en la actualidad, reguladas específicamente en la LOMCE (Ley Orgánica para la Mejora de la Calidad Educativa) del año 2013. Pero no es una situación novedosa, ya que anteriormente en la LOE (Ley Orgánica de Educación) del año 2006 se hacía una primera prescripción obligatoria para todos los centros educativos en este sentido. Se alude, por tanto, a un aprendizaje que se promueve en las escuelas y el conjunto del sistema educativo desde hace más de 10 años. Sin embargo, esta competencia sigue precisando de nuestra atención, debido a que investigaciones previas realizadas muestran dificultades en su adquisición (Alonso-Ferreiro y Gewerc, 2018; Area et al., 2014). Tanto las orientaciones desarrolladas para su definición como la delimitación de lo que supone el desarrollo sólido de esta competencia en la actualidad, ponen en entredicho la realidad que aparentemente se supone en el alumnado que finaliza la Educación Primaria (AlonsoFerreiro y Gewerc, 2018).

Esta investigación parte de una concepción amplia en la adquisición de la competencia, superando una perspectiva estrictamente escolar. Es por ello que se integra a la familia, pero también otros espacios sociales que puedan ayudar en el desarrollo de la competencia, como puede ser el grupo de iguales. Para abordar este campo, se han revisado diferentes constructos en relación con la CD, ya sea Digital Literacy (Bawden, 2008; Knobel y Lankshear, 2008), las habilidades digitales (Van Deursen y Van Dijk, 2014) o la competencia mediática (Buckingham, 2007; Jenkins, 2009). La visión de Ferrari (2013) sobre la CD ha sido uno de los principales puntos de apoyo utilizados en esta investigación para comprender sus posibilidades. Esta perspectiva implica el desarrollo de una serie de habilidades, estrategias y conocimientos con tecnologías digitales para poder, por ejemplo, localizar información, procesarla y generar conocimiento propio en múltiples formatos. Esta realidad supone un desafío para los espacios escolares, ya que este tipo de planteamiento implica su integración de forma estratégica en el lugar donde se desarrolla la enseñanza y el aprendizaje. La realidad actual exige la adecuación de las propuestas escolares debido a los nuevos requerimientos, en los que las tecnologías digitales se han convertido en elementos claves para su desarrollo. La gestión de estas tecnologías se ha revelado como fundamental para la construcción del conocimiento y, en este sentido, los retos se acumulan. Ya no solo se trata de acceder a la información, sino de contrastarla adecuadamente empleando habilidades cada vez más complejas para la gestión de su veracidad. Por otra parte, su procesamiento y tratamiento (en forma de conocimiento elaborado por los propios sujetos en múltiples lenguajes digitales), requiere del dominio de herramientas y espacios que pone en entredicho el aprendizaje del alumnado de las etapas obligatorias:

Aprendizajes ausentes en la Competencia Digital de preadolescentes: un estudio de casos pertenecientes a contextos socioculturales desfavorables. Fernando Fraga-Varela, Esther VilaCouñago y Eulogio Pernas-Morado.

Página 3 de 18 
El lenguaje iconográfico y audiovisual está inundando los procesos comunicativos de la Red y ello requiere la alfabetización de los sujetos, tanto como consumidores de este tipo de productos como en su formación como emisores, de forma que posean las competencias expresivas para expresarse con los formatos multimedia y los lenguajes audiovisuales (Area y Pessoa, 2012, pp. 16-17).

En este sentido, el Proyecto DIGCOMP de Ferrari (2013) y posteriores revisiones (Vuorikari, Punie, Carretero y Van den Brande, 2016) consiguen dar cuenta de los conocimientos, actitudes y habilidades a través de su propuesta de cinco dimensiones: 1) Información y Alfabetización Informacional, 2) Comunicación y Colaboración, 3) Creación de Contenido Digital, 4) Seguridad y 5) Resolución de problemas; junto con un tratamiento sistemático de las mismas en múltiples subapartados a través de veintiuna competencias.

\section{Objetivos}

El trabajo que se expone forma parte del Proyecto de Investigación "Competencia digital en estudiantes de educación obligatoria. Entornos sociofamiliares, procesos de apropiación y propuestas de e-inclusión" (CDEPI) (EDU2015-67975-C3-1-P), que tiene su foco en el estudio de la CD en alumnado de Educación Primaria. El proyecto CDEPI se acota precisamente al último año de escolarización de esta etapa, $6^{\circ}$ curso, por considerar que es donde el desarrollo de la competencia podría presuponerse relativamente avanzado. Su objetivo principal es la identificación, análisis, evaluación y comprensión de la denominada CD.

Más concretamente, la pretensión del estudio es identificar indicadores relevantes de niños y niñas en edades de 12 años residentes en Galicia -que sirvan como elementos de referencia para la construcción de un instrumento de evaluación de la CD (siguiente etapa del proyecto)- y analizar las relaciones que se dan entre el papel de las familias (su extracción social y cultural), de otros agentes educativos y del grupo de iguales respecto al desarrollo de su CD. Así mismo, se pretende comprender la influencia de este aprendizaje en los procesos de inclusión social y formular algunas consideraciones a nivel escolar y social de cara a la mejora de la CD del alumnado.

Como objetivo específico del presente trabajo se pone el foco en casos de alumnos de bajo nivel sociocultural y económico. Se pretende indagar acerca de las repercusiones que tiene esta configuración en el desarrollo de la CD ante circunstancias familiares adversas en el ambiente familiar.

\section{Método}

Para dar respuesta a los objetivos formulados, se optó por una metodología mixta de investigación. Este tipo de propuesta supone utilizar métodos, técnicas o datos cuantitativos y cualitativos en el marco del mismo estudio. En particular, se empleó un diseño de tipo secuencial explicativo, el cual combina una primera fase cuantitativa y una segunda fase cualitativa (Creswell y Plano, 2018). Concretamente en este estudio, en la primera fase se empleó el método de encuesta, cuyos resultados se utilizaron para llevar a cabo la segunda y principal fase de la investigación: un estudio de casos. Además, atendiendo a la función que cumple la parte cuantitativa del estudio, conviene señalar que este responde a una variante del diseño secuencial explicativo denominada "selección de casos" (Creswell y Plano, 2018, p. 82). Como se presenta en la Figura 1, en una primera

Aprendizajes ausentes en la Competencia Digital de preadolescentes: un estudio de casos pertenecientes a contextos socioculturales desfavorables. Fernando Fraga-Varela, Esther VilaCouñago y Eulogio Pernas-Morado.

Página 4 de 18 
fase se recogieron datos de una muestra amplia de sujetos y, a partir de los resultados obtenidos, se seleccionaron los participantes del estudio de casos.

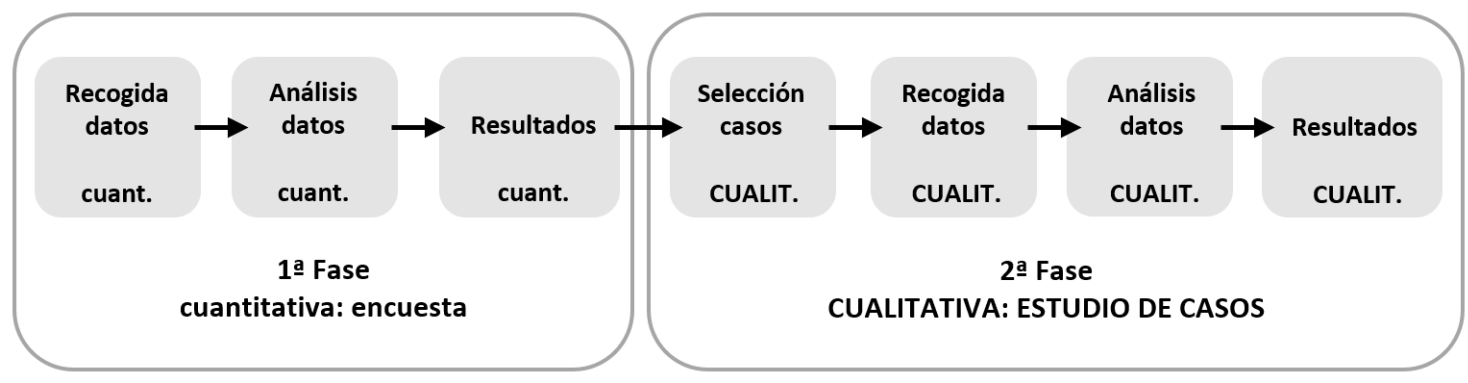

Figura 1. Diseño mixto explicativo de selección de casos

\section{Estudio cuantitativo para la selección de los casos}

Acudimos a centros educativos públicos de la Comunidad Autónoma de Galicia inmersos en proyectos de saturación tecnológica con el modelo 1 a 1 , en los que se aplicó un cuestionario elaborado ad hoc y dirigido a las familias del alumnado de $6^{\circ}$ curso de Educación Primaria. Como criterios fundamentales para la selección de la muestra, atendimos a la representatividad de los diferentes entornos socio-culturales y económicos - dada la importancia que podía tener esta variable en el proceso de construcción de la CD-, y a la accesibilidad de los propios sujetos (que se prestaran a la realización de la investigación).

El cuestionario nos permitió conocer algunas condiciones del entorno socio-familiar a partir de determinados indicadores como el número y tipo de dispositivos tecnológicos existentes en el hogar, los estudios y la profesión de los progenitores y la concesión o no de ayudas para material escolar. El instrumento fue previamente validado por un conjunto de expertos en TIC y en metodología de investigación. Constaba de cuatro partes: la primera se centraba en las características sociodemográficas del niño/a; la segunda versaba sobre los usos de la tecnología en el hogar; la tercera ahondaba en los hábitos del niño/a y en las actividades realizadas fuera del horario escolar; y una cuarta parte preguntaba por datos familiares, donde se incluían las cuestiones relativas a la ocupación y estudios de los progenitores, solicitud de ayudas, etc.

Se recogió información de un total de 182 familias pertenecientes a cinco centros educativos diferentes. Tras el análisis de las respuestas dadas, se preseleccionaron -atendiendo a la "máxima rentabilidad" para responder a nuestros objetivos (Stake, 2010, p. 17) - un grupo de niños y niñas pertenecientes a distintos centros y a entornos familiares con distinto capital cultural. A través del centro educativo se comunicó a las familias la selección realizada y se les invitó a participar en la investigación, garantizando el principio de consentimiento informado en relación con los objetivos del estudio, el tipo de participación solicitada, la fuente de financiación del proyecto y los posibles usos de los resultados derivados.

\section{Estudio de casos como método principal}

Se optó por el método de estudio de casos por ser particularmente idóneo para comprender en profundidad un fenómeno de la vida real y para responder a interrogantes marcados

Aprendizajes ausentes en la Competencia Digital de preadolescentes: un estudio de casos pertenecientes a contextos socioculturales desfavorables. Fernando Fraga-Varela, Esther VilaCouñago y Eulogio Pernas-Morado.

Página 5 de 18 
por el "cómo" y el "por qué", especialmente cuando tal comprensión entraña importantes condiciones contextuales (Yin, 2009). Concretamente, se llevó a cabo un estudio de casos múltiple - con varios casos que se asemejan y difieren entre sí- de tipo analítico (Coller, 2005), pues este no trata sólo de detectar y descubrir un fenómeno, sino que va más allá buscando su comprensión y posibles relaciones con otros; y de diseño holístico, dado que se examina la naturaleza global del fenómeno, entendiendo que cada sujeto ilustra una unidad de análisis (Yin, 2009).

El estudio estuvo conformado por un total de seis sujetos: dos pertenecientes a familias de capital cultural alto (dos hermanas), dos de medio (dos hermanos) y dos de bajo (dos niños). Justamente en estos últimos casos de capital cultural bajo se basa el presente artículo. A los protagonistas los llamamos Bieito y Jaime -nombres ficticios- con el objetivo de dar verosimilitud al trabajo y respetar su anonimato.

Fueron dos los investigadores dedicados al estudio de cada caso y se utilizaron diversas técnicas de recogida de datos, siendo, además, aplicadas a informantes diferentes. En concreto, empleamos:

- Entrevistas en profundidad a los niños, a sus progenitores o tutores legales -la abuela en el caso de Bieito y la madre en el caso de Jaime-, al profesorado-tutor y al responsable de la dirección del centro educativo.

- Observación participante de los sujetos, fundamentalmente de los comportamientos de los niños en su actuación ante las tecnologías. En estas sesiones realizaron tareas o jugaron a distintas aplicaciones con su ordenador portátil o su tableta que fueron grabadas para su análisis posterior.

- Observación no participante del entorno escolar.

- Diarios de campo del entrevistador.

- Diarios de los niños. Cada caso elaboró un diario en donde recogió las actividades diarias en las que utilizaba las tecnologías, pudiendo elegir el formato que considerase más apropiado (audio-texto-imagen fija o video) y también el medio por el cual se lo enviaba al investigador.

- Especificación de la PLN de los niños, a través de la cual ubicaron su núcleo de relaciones.

- Análisis de documentos del centro escolar al que asisten los niños.

La triangulación de fuentes y técnicas, así como de personal investigador, permitió obtener información más rica, verla desde diferentes ángulos, profundizar en la realidad de los casos y contribuir a una explicación válida de la CD de los sujetos.

La información recogida se analizó atendiendo a las pautas de la teoría fundamentada (Glaser y Strauss, 1967). Primeramente se realizó una codificación abierta del material recopilado de cada uno de los casos, en la que el texto fue dividido en porciones de información con significado a los que se les asignó un código, utilizando un procedimiento inductivo. Posteriormente los códigos fueron agrupados en categorías, en base a una temática similar. El segundo paso consistió en llevar a cabo la codificación axial, que se centró en establecer relaciones entre categorías y subcategorías, para lo que buscamos claves y patrones emergentes en el texto que permitieran relacionar las categorías entre sí. Finalmente se llegó a la codificación selectiva, determinando las categorías más importantes e integrando proposiciones para lograr una comprensión general -una teoría- de los casos, permitiéndonos a la vez comparar las similitudes y diferencias existentes entre estos. Todo este proceso de análisis se realizó con la ayuda

Aprendizajes ausentes en la Competencia Digital de preadolescentes: un estudio de casos pertenecientes a contextos socioculturales desfavorables. Fernando Fraga-Varela, Esther VilaCouñago y Eulogio Pernas-Morado.

Página 6 de 18 
del programa Atlas.ti 7, que facilitó la organización, el almacenamiento, el etiquetaje y la recuperación de la información, además del establecimiento de conexiones entre códigos y la visualización de dichas relaciones en mapas (Muñoz y Sahagún, 2017).

Para finalizar el proceso de análisis, hicimos llegar a los participantes un informe con los principales hallazgos, que ha contribuido a la validación de las observaciones e interpretaciones propuestas. Esta es una cuestión que entendemos de máxima prioridad hacia los verdaderos implicados y destinatarios del trabajo de investigación, tal y como nos indica Tójar (2006, p. 348): "Tienen derecho a participar de las conclusiones, a dar su opinión, a debatir los resultados con los investigadores o investigadoras".

En la exposición de los resultados que sigue a continuación, se señalan citas textuales de los participantes, identificadas a través de "Bi" para Bieito y "Ja" para Jaime, además de ir acompañadas de un código que designa a las demás personas entrevistadas: "TU" para tutor/a, "DIR" para director, "AB" para abuela y "M" para madre.

\section{Resultados}

Los resultados obtenidos serán presentados en este apartado atendiendo a una contextualización con datos clave de cada caso, que permiten comprender el entorno en el que crecen ambos protagonistas. Posteriormente, se presenta un apartado en el que se abordan los elementos de la CD que aparecen tras el análisis, tratando tanto la presencia como la ausencia de las diferentes dimensiones que la componen profundizando en ejemplos observados y en los entornos donde se adquieren estas habilidades, conocimientos y actitudes.

\section{El caso Bieito: viejas normas para nuevas tecnologías}

Bieito nace en un contexto familiar, social y económico difícil. Su madre se encuentra fuera de Galicia y su padre en el extranjero. Fruto del divorcio, y sobre todo a partir del nivel de la desestructuración familiar que se desarrolla a lo largo de los años, desde los servicios sociales se traslada su tutela a los abuelos maternos junto a sus dos hermanas. Así, abuelos y nietos conviven en el extrarradio de la ciudad, en un contexto difuso entre lo rural y lo urbano.

El protagonista se desarrolla en dos espacios de referencia: la casa de sus abuelos maternos y la escuela. El análisis de su entorno personal, conformado por amistades o vecinos, no se ha dado debido a la mínima proyección de su socialización en entornos diferentes a la escuela y el hogar. Esta realidad es consecuencia, en parte, de una protección de los abuelos sobre el niño para evitar cualquier problema con los servicios sociales. La familia tiene serias dificultades económicas para poder pagarle actividades extraescolares: "Porque cuesta mucho dinero" (Bi). También porque tienen limitaciones para desplazarse debido a la situación de la vivienda frente a la ubicación de las actividades, como el fútbol, y esto condiciona su participación.

Destacan desde un inicio aspectos que se vuelven ejes clave para la comprensión de la forma en la que Bieito desarrolla sus competencias en este ámbito: por un lado, el consumo indiscriminado y sin ningún tipo de control de los medios presentes en el ámbito familiar y, por otro, la traslación de estas formas de apropiación a las tecnologías digitales disponibles en su entorno. La televisión es un elemento omnipresente en la vida de Bieito sin ningún tipo de regulación. Él mismo reconoce que ve la TV "todo el día" (Bi), con una total libertad respecto al contenido "veo lo que quiero" (Bi). Hubo un ordenador,

Aprendizajes ausentes en la Competencia Digital de preadolescentes: un estudio de casos pertenecientes a contextos socioculturales desfavorables. Fernando Fraga-Varela, Esther VilaCouñago y Eulogio Pernas-Morado.

Página 7 de 18 
ahora estropeado, y no hay una aparente necesidad de reemplazarlo: "No, no tenemos" (Bi). En este contexto irrumpe un dispositivo, todos tienen móvil, y Bieito, por supuesto, también posee uno con número propio y conexión a internet. El móvil de Bieito le llega a través de un mecanismo que se ha descubierto gracias a este caso y que hemos denominado en el proyecto "herencia digital": un adulto de su entorno próximo que cambia de móvil y que es heredado por hijos/as o sobrinos/as. "Se lo dio su... su tío, su padrino" (Bi_AB). Bieito ni siquiera llega a pedirlo. "Entrevistadora: ¡Ah!, entonces Bieito no pedía el móvil. María: No, no.” (Bi_AB). El mecanismo de herencia digital también ha posibilitado que juegue en otros dispositivos, como la PSP.

Su hermana mayor, que se encuentra estudiando en la universidad, interviene respecto al control de acceso (como también lo hace con su hermana mediana) y al uso del dispositivo. Aunque hay elementos comunes a la televisión, ya que su regulación es aparentemente laxa, sí se diferencia en aspectos concretos. Es el caso de las redes sociales, como Instagram, claramente monitoreada por su hermana mayor. Sin embargo, no hay ningún tipo de orientación que vaya más allá. Bieito juega a cualquier juego disponible en las tiendas de aplicaciones sin llegar a sondear apps o aplicaciones que exploren de forma más contundente las posibilidades de la CD. Esto se evidencia cuando dice tener cuenta de correo pero de los antiguos usuarios, por ese mecanismo de herencia digital, ya que el móvil le llega sin reiniciar. Las cuentas de correo y redes sociales están a su disposición con perfiles que no son suyos: "Bieito: también tengo, pero es oscar@gmail.com. Entrevistadora: Y, y, ¿por qué eres Óscar? Bieito: Porque mi prima le dejó el móvil a una persona" (Bi). En otros casos se trata de perfiles ya en desuso, de redes sociales que han desaparecido.

La familia supervisa e interviene donde tiene conocimiento de un riesgo potencial sobre el niño. La televisión es inocua a ojos de los abuelos, también para su hermana mayor, porque saber que está físicamente en casa es una garantía aparente de seguridad en su visión de la realidad del niño. Sin embargo, para su hermana mayor, el móvil y las redes sociales sí necesitan un mayor control, y ahí ella interviene y marca la pauta en el hogar, al igual que los abuelos que controlan los tiempos de uso del móvil. Sin embargo, no se aprecia peligro en el uso de juegos u otras aplicaciones disponibles.

\section{El caso Jaime: conectado a lo virtual, desconectado de lo real}

A principios de la investigación, Jaime residía con su madre, con su padre y con su hermana pequeña de 8 años. En los meses en los que se realizó la primera recogida de datos, sin embargo, su entorno familiar sufrió importantes cambios debido al divorcio de sus progenitores y al abandono del domicilio familiar por parte del padre. Tanto la madre como el padre son jóvenes, alrededor de 30 años, que tuvieron que hacer frente a su situación/condición de paternidad cuando aún eran menores de edad. La madre es empleada de hogar y trabaja por las mañanas. El padre se encuentra en situación de desempleo. La situación socioeconómica familiar es, por tanto, complicada.

El padre compartía tiempos y espacios con Jaime a la hora de interactuar con las tecnologías, sobre todo en el uso de videojuegos. El niño pasaba las tardes en el "búnker", tal como lo denomina su madre, para referirse a una habitación rodeada de dispositivos tecnológicos y con estanterías cargadas de juegos. La marcha del padre del domicilio familiar supuso la ruptura de toda esta dinámica paterno-filial, al cortar la conexión de internet y llevarse consigo la consola.

Aprendizajes ausentes en la Competencia Digital de preadolescentes: un estudio de casos pertenecientes a contextos socioculturales desfavorables. Fernando Fraga-Varela, Esther VilaCouñago y Eulogio Pernas-Morado.

Página 8 de 18 
Por su parte, la madre - a la que llamamos Natividad- es la principal responsable del cuidado de los hijos. Cabe destacar que Jaime está diagnosticado de Trastorno por Déficit de Atención e Hiperactividad (TDAH) y la madre pone de manifiesto que su hijo la requiere para todo, excepto para aquello que concierne a los videojuegos.

Por lo que respecta a la tecnología, el primer contacto de Jaime tuvo lugar a una edad muy temprana: "Desde que tenía tres meses. Mi padre me daba el mando sin conectar, y yo jugaba, hacía que jugaba" (Ja). Con pocos años ya tenía un dominio total de la videoconsola: "Yo creo que con cinco años ya jugaba con su padre perfectamente a la consola" (Ja_M).

Jaime empezó a manejar la tablet con nueve años. En cuanto al teléfono móvil, aunque aún no posee uno propio, ha quedado patente que desde muy pequeño tuvo acceso a los de sus familiares, teniendo en cuenta que usaban este dispositivo para mostrarle vídeos: "Los vídeos en el móvil, hubo una temporada, cuando era más pequeño, que lo que le gusta... Veía lo que es vídeos en inglés de los dibujos, y se los poníamos en inglés" (Ja_M). Como último dispositivo con el que se familiarizó, Jaime hace mención al ordenador de sobremesa. Señala que su contacto con el ordenador es relativamente reciente. Este se produjo en casa de su tía, antes que en el colegio. Su uso se ciñó -y aún sigue siendo así en la actualidad- a juegos de PC (sin conexión a internet).

Jaime hace un uso muy frecuente de YouTube en su tiempo de ocio. El uso de esta plataforma va más allá de ser un mero espectador. Jaime habla de un canal propio que tienen su padre y él de forma compartida en el que cuelgan vídeos jugando a videojuegos. Su padre también es youtuber y, al parecer, le dedica mucho tiempo a la grabación y edición de vídeos del tipo conocido como gameplays (en los que un jugador graba una partida a un videojuego para que se aprecie como ha jugado). Jaime llega a hablar de ese canal como propio, al que están suscritos algunos de sus compañeros/as de clase y quienes, a veces, le dejan comentarios en sus vídeos.

En cuanto a los videojuegos propiamente dichos, el niño muestra una clara preferencia hacia aquellos que están en formato App, como Clash Royale, y de acción y guerra, muchos de ellos no recomendados para menores de 18 años (entre otras razones porque contienen violencia implícita).

La madre de Jaime percibe los videojuegos como una oportunidad para su hijo con TDAH, en cuanto logran mantenerlo concentrado y tranquilo durante el tiempo que está utilizándolos; a diferencia, por ejemplo, de la televisión, a la que no presta mucha atención ni tampoco para quieto durante el tiempo que la ve. Los videojuegos consiguen esa gran motivación que necesita para estar centrado en la tarea. Además, Natividad destaca el mayor grado de autonomía que muestra Jaime en el uso de los videojuegos, y en el mundo tecnológico en general, en contraste con otras actividades cotidianas: "En ese tipo de mundo es muy independiente. Yo creo que también es porque le llama tanto. Cómo es algo que él sabe hacer y nosotros no: "Aquí es mi terreno... Y aquí me muevo yo"(Ja_M). La peculiar red de amistades de Jaime es otro de los aspectos llamativos de este caso. En el barrio no tiene lazos de amistad; "con los amigos del cole no tiene relación fuera del cole" (Ja_M) y con los amigos del entorno digital mantiene un contacto diario, pero, como comenta el propio Jaime: "No los conozco en la vida real" (Ja). Es decir, el niño no cuenta con un grupo de amigos consolidado y son los colegas del entorno virtual, junto con otros usuarios desconocidos, los que conforman su red personal de aprendizaje (PLN).

Aprendizajes ausentes en la Competencia Digital de preadolescentes: un estudio de casos pertenecientes a contextos socioculturales desfavorables. Fernando Fraga-Varela, Esther VilaCouñago y Eulogio Pernas-Morado.

Página 9 de 18 


\section{La escuela: un mismo uniforme para dos perfiles diferentes}

El análisis de la realidad en la escuela se realiza a través de entrevistas al tutor de Bieito, la tutora de Jaime y al director, cruzándose con observaciones y entrevistas de los casos. La situación revela elementos fundamentales para comprender la realidad desde dentro de la escuela y cómo ésta hace frente a las demandas de lo que supone el trabajo competencial en la actualidad, en concreto sobre la $\mathrm{CD}$. Conviene tener presente que nos encontramos ante una de las 7 competencias clave que se deben abordar en los entornos de escolarización obligatoria. Por lo tanto, la realidad de la CD pasa por comprender la situación de conjunto.

El centro al que asisten Bieito y Jaime se caracteriza por estar ubicado en una zona urbana. Se trata de un colegio de dos líneas, con 27 alumnos por aula (máximo legal) al que se suman los repetidores. Además, se trata de un centro adscrito al proyecto Abalar de la Xunta de Galicia, que dotaba de ordenadores y portátiles a $5^{\circ}$ y $6^{\circ}$ de Educación Primaria (una adaptación del Plan Escuela 2.0 estatal). En el momento de las entrevistas el director se encuentra en el ecuador de su gestión. Describe una relación muy cercana con las familias, conocedor de sus circunstancias.

Señala la biografía de Bieito como una realidad muy dura para el niño, reforzando los hallazgos de las entrevistas y observaciones: "Pasa de un centro de acogida a normalizar su situación en un centro, pasa de una adaptación curricular a no tenerla, pasa de tener una... Una... Una realidad familiar volcada en su abuela y sus... sus hermanas" (DIR). Señala con preocupación el futuro de Bieito. También su tutor muestra su preocupación al respecto: "O sea, el futuro de cualquier niño a largo plazo está más o menos pautado y dirigido y tal, y el de Bieito no. O sea, el de Bieito depende de muchísimos factores, es que tampoco tienen un arraigo familiar como una familia tradicional" (Bi_TU).

Centrándonos en el caso de Jaime, y como señala su tutora, hay una gran brecha entre las actividades con tecnologías que desarrolla en el hogar y las que desenvuelve en la escuela. Mientras que en el aula el uso de la tecnología se reduce sobre todo a la búsqueda de información a través de un ordenador que actualmente parece anticuado, en casa busca información, se comunica con otros usuarios y crea contenido digital. Por una parte, la propia tutora manifiesta una visión desfavorable sobre la repercusión de los videojuegos en el caso de Jaime, describiendo que acrecientan sus problemas académicos. En su opinión, el gran uso que realiza de los videojuegos está repercutiendo negativamente en su rendimiento al no dedicar el tiempo necesario a estudiar y a realizar las tareas encomendadas para casa: "¿Qué esperamos? Que un niño que le dedica su vida, su vida, porque es su vida, a eso, no va a tener como mínimo déficit de atención?” (Ja_TU). Por otra parte, la madre impide el contacto del niño a través de la cuenta de Gmail que emplea la docente y el grupo de compañeros y compañeras de clase. Aquí se entremezclan motivos como la mala relación entre ellas, el hecho de no ser un recurso propio de la Consellería de Educación y el uso de esta cuenta por parte de la profesora -según la madre de Jaime- exclusivamente para "poner trabajos que ella quiere" (Ja_M). Así, la madre no favorece tampoco el uso del ordenador en el hogar para la realización de las tareas escolares.

La realidad constatada a lo largo de la investigación de los dos casos no parece mostrar una actuación coordinada y coherente entre escuela y familia en sus formulaciones sobre el desarrollo de la CD, ya que en ambas familias la colaboración se torna complicada y se evidencian desconexiones explícitas y falta de retroalimentación. Cada uno avanza de

Aprendizajes ausentes en la Competencia Digital de preadolescentes: un estudio de casos pertenecientes a contextos socioculturales desfavorables. Fernando Fraga-Varela, Esther VilaCouñago y Eulogio Pernas-Morado. 
forma desacompasada, generando una dinámica específica en función del lugar donde le corresponde estar a lo largo del día y de la semana.

\section{Docentes y dirección ante distintos focos}

A pesar de que el centro cuenta con el programa Abalar, el uso del portátil, por ejemplo, y las actividades con tecnología son muy limitadas debido, en parte, a conflictos durante procesos de innovación activados anteriormente. Las familias reclamaban un lugar central en la escuela para el libro de texto, presentando resistencia ante cualquier proceso que supusiese su ausencia. Su presencia reforzaba un contrato no escrito en el proceso de aprendizaje, y una garantía para que las familias pudiesen acompañar a sus hijos en las tareas propuestas. A esta realidad se añade una excesiva ratio por aula y la diferencia significativa entre niveles de desarrollo competencial del alumnado. Así, el profesorado se limita a considerar un "nivel medio" general para todos, de igual modo que a utilizar un mismo libro, en cada materia, para el conjunto de aula. En este contexto, las individualidades se diluyen, no hay un seguimiento que permita apoyar la situación de desprotección particular de Bieito o Jaime: "Cuando las cosas van parejas pues todo el mundo va bien, pero como lo intentes trabajar en diferentes ritmos, o en diferentes cosas... eh..." (Bi_TU). Encajar en este contexto el uso de dispositivos digitales como Abalar, es complejo. Se plantean estrategias coherentes con el día a día de procesos claramente estructurados por la lógica de las editoriales y la máxima de "todos a una".

Juan, el director del colegio, señala además la necesidad de que cada centro apueste por una "dirección" que lo distinga de otros: "Es descomunal, porque yo suelo entrar en la web de la Consellería de Educación y ver todo lo que está activo en paralelo, hay muchas iniciativas [...] Es un poco menú de restaurante. Tú llegas, y qué ¿tú qué quieres? Entonces tú configuras tu menú. [...]". Subraya el exceso de demandas que tiene un centro "o sea tienes cincuenta mil cosas, o sea, si diariamente lees el DOG [Diario Oficial de Galicia] te das cuenta de que es imposible... Seguir eso" (DIR). En este juego se indica que actualmente han apostado en esa optatividad en torno a una línea concreta: "Estamos ahora en el programa de introducción a la robótica" (DIR), pero apunta también una falta de formación del profesorado en la que él se incluye para poder llevar a buen término la iniciativa; hasta el punto de reconocer la posibilidad de quedar en evidencia ante el propio alumnado y familias.

En este contexto el profesorado acompaña la situación de Bieito y Jaime con la mejor de sus intenciones, pero siendo consciente de las limitaciones que la situación presenta a la luz de las necesidades de un contexto familiar y personal tan complejo.

\section{Diferentemente iguales: principales hallazgos}

Se presenta a continuación una síntesis de los principales hallazgos del estudio en ambos casos (Bieito en la Tabla 1 y Jaime en la Tabla 2), incidiendo en la relación entre el desarrollo de la CD y los dos "escenarios" analizados: hogar y escuela. Se ha descartado el trabajo con el grupo de iguales porque ni Bieito ni Jaime presentan elementos significativos en lo que se refiere a este ámbito.

Las diferentes dimensiones señaladas en el proyecto DIGCOMP son reflejadas en este apartado. Las sesiones de observación y entrevista con los dos protagonistas de esta historia permiten desvelar no sólo su autopercepción, sino ejemplificaciones y muestras de habilidades competenciales en contextos reales avanzando en un conocimiento real de

Aprendizajes ausentes en la Competencia Digital de preadolescentes: un estudio de casos pertenecientes a contextos socioculturales desfavorables. Fernando Fraga-Varela, Esther VilaCouñago y Eulogio Pernas-Morado. 
lo que supone para cada caso. Se añaden ejemplos concretos que ilustran cada una de las dimensiones de la $\mathrm{CD}$ en relación con los escenarios en los que se desarrollan.

Tabla 1. La relación entre las dimensiones de la CD y los dos escenarios para el caso de Bieito

\begin{tabular}{|c|c|c|c|}
\hline $\begin{array}{c}\text { DIMENSIÓN } \\
\text { CD }\end{array}$ & OBSERVACIÓN CON BIEITO & AUTOPERCEPCIÓN BIEITO & ESCENARIO \\
\hline $\begin{array}{l}\text { Información y } \\
\text { Alfabetización } \\
\text { Informacional }\end{array}$ & $\begin{array}{l}\text { Búsqueda de información en } \\
\text { Google, de forma básica. Añade } \\
\text { conceptos fundamentales en la } \\
\text { búsqueda. La selección del enlace } \\
\text { que realiza es el primero, sin leer } \\
\text { otros resultados o la información } \\
\text { básica que aparece ahí. No hay } \\
\text { evaluación de los datos. }\end{array}$ & $\begin{array}{l}\text { Señala búsqueda con frecuencia } \\
\text { de elementos audiovisuales } \\
\text { (principalmente vídeos en } \\
\text { YouTube) y otro tipo de } \\
\text { información para tareas en el } \\
\text { aula. }\end{array}$ & $\begin{array}{l}\text { HOGAR } \\
\text { ESCUELA }\end{array}$ \\
\hline $\begin{array}{l}\text { Comunicación y } \\
\text { Colaboración }\end{array}$ & $\begin{array}{l}\text { Se le facilita la aplicación de } \\
\text { WhatsApp por ser la que indica } \\
\text { como la única que utiliza. Muestra } \\
\text { manejo con bastante soltura. Es la } \\
\text { única herramienta que utiliza para la } \\
\text { comunicación. }\end{array}$ & $\begin{array}{l}\text { WhatsApp es la herramienta que } \\
\text { de forma preferente considera } \\
\text { para comunicarse con sus padres, } \\
\text { que viven alejados y con algunos } \\
\text { compañeros del colegio. Se siente } \\
\text { hábil en su uso pero no gestiona } \\
\text { su identidad digital. }\end{array}$ & HOGAR \\
\hline $\begin{array}{l}\text { Creación de } \\
\text { Contenido } \\
\text { Digital }\end{array}$ & $\begin{array}{l}\text { La observación pone de relieve poco } \\
\text { conocimiento sobre la posición de } \\
\text { las teclas en el teclado, además del } \\
\text { desconocimiento sobre algún } \\
\text { software editor de texto y/o de } \\
\text { imagen. No muestra habilidad en la } \\
\text { utilización de software de creación. }\end{array}$ & $\begin{array}{l}\text { A cuestiones sobre si utiliza } \\
\text { algún programa para escribir } \\
\text { trabajos o para dibujar, etc. } \\
\text { responde que sí, pero no sabe } \\
\text { identificar ninguno con nombres. }\end{array}$ & (AUSENCIA) \\
\hline Seguridad & $\begin{array}{l}\text { Mientras realiza la búsqueda en } \\
\text { Google y clica en el primer enlace } \\
\text { de lo que busca, aparece una pestaña } \\
\text { con publicidad sobre un hotel con } \\
\text { spa y en ese momento en voz alta } \\
\text { señala que va a comprarlo para su } \\
\text { hermana y se dispone a abrir otra } \\
\text { pestaña. No lee. }\end{array}$ & $\begin{array}{l}\text { No tiene cuentas digitales } \\
\text { propias, no están a su nombre: } \\
\text { Gmail y otras redes sociales. No } \\
\text { parece consciente de las } \\
\text { consecuencias de este hecho ni de } \\
\text { las normas para darse de alta en } \\
\text { las diferentes plataformas. }\end{array}$ & (AUSENCIA) \\
\hline $\begin{array}{l}\text { Resolución de } \\
\text { Problemas }\end{array}$ & $\begin{array}{l}\text { No se muestra hábil en rutinas de } \\
\text { trabajo que se salgan de los } \\
\text { procedimientos de aula. }\end{array}$ & $\begin{array}{l}\text { En su discurso afirma poseer } \\
\text { habilidades en el manejo de todas } \\
\text { las herramientas que se } \\
\text { comentan. No siente } \\
\text { desconocimiento o lagunas. Tiene } \\
\text { un discurso elaborado sobre los } \\
\text { últimos dispositivos de moda. }\end{array}$ & (AUSENCIA) \\
\hline
\end{tabular}

Aprendizajes ausentes en la Competencia Digital de preadolescentes: un estudio de casos pertenecientes a contextos socioculturales desfavorables. Fernando Fraga-Varela, Esther VilaCouñago y Eulogio Pernas-Morado. 
RED. Revista de Educación a Distancia. Núm. 61, Artíc. 04, 30-10-2019

DOI: http://dx.doi.org/10.6018/red/61/04

Tabla 2. La relación entre las dimensiones de la CD y los dos escenarios para el caso de Jaime

\begin{tabular}{|c|c|c|c|}
\hline $\begin{array}{l}\text { DIMENSIÓN } \\
\text { CD }\end{array}$ & OBSERVACIÓN CON JAIME & $\begin{array}{l}\text { AUTOPERCEPCIÓN } \\
\text { JAIME }\end{array}$ & ESCENARIO \\
\hline $\begin{array}{l}\text { Información y } \\
\text { Alfabetización } \\
\text { Informacional }\end{array}$ & $\begin{array}{l}\text { Gran habilidad instrumental, sobre todo con la } \\
\text { tablet: selecciona la opción wifi para buscar una } \\
\text { conexión, ajusta el brillo de la pantalla o } \\
\text { comprueba el tiempo de la batería (con los } \\
\text { videojuegos es más hábil aún). Con el ordenador } \\
\text { no es tan hábil, pero sabe usar el ratón o el } \\
\text { navegador, desplazarse por las pestañas, copiar y } \\
\text { pegar o abrir un programa. Con el móvil es } \\
\text { menos seguro, le cuesta ubicar las letras del } \\
\text { teclado. Sabe encontrar información con } \\
\text { diferentes motores de búsqueda; usa palabras } \\
\text { clave, etc., pero no analiza/interpreta la } \\
\text { información hallada, ni coteja las fuentes } \\
\text { consultadas. Se suele quedar con la información } \\
\text { de una única página (Wikipedia). }\end{array}$ & $\begin{array}{l}\text { Alta percepción de sus } \\
\text { habilidades } \\
\text { instrumentales, en } \\
\text { especial con los } \\
\text { videojuegos, pero } \\
\text { también con la tablet. } \\
\text { Del ordenador no tiene la } \\
\text { misma consideración } \\
\text { ("porque nunca lo uso", } \\
\text { Ja). } \\
\text { También valora } \\
\text { positivamente su } \\
\text { capacidad para buscar } \\
\text { información (en especial } \\
\text { respecto a sus } \\
\text { compañeros y } \\
\text { compañeras de clase). }\end{array}$ & $\begin{array}{l}\text { HOGAR } \\
\text { ESCUELA }\end{array}$ \\
\hline $\begin{array}{l}\text { Comunicación } \\
\text { y } \\
\text { Colaboración }\end{array}$ & $\begin{array}{l}\text { Con las consolas, al jugar en modo multijugador } \\
\text { online usa el micrófono para comunicarse } \\
\text { durante las partidas con amigos virtuales y } \\
\text { usuarios desconocidos. También se comunica } \\
\text { con los amigos virtuales a través de los grupos } \\
\text { que crea específicamente para ello. En la tablet, } \\
\text { usa las opciones de "clan" de Clash Royale para } \\
\text { charlar o colaborar con su padre, su hermana y } \\
\text { otros jugadores. Hace comentarios en vídeos de } \\
\text { gamers en YouTube. } \\
\text { Sin embargo, no participa en el grupo creado por } \\
\text { la profesora a través de la cuenta de Gmail ni } \\
\text { deja comentarios en el blog de aula. }\end{array}$ & $\begin{array}{l}\text { Le da mucha importancia } \\
\text { a la retroalimentación } \\
\text { que recibe de su padre. } \\
\text { Le gusta compartir sus } \\
\text { creaciones virtuales } \\
\text { (realizadas con el editor } \\
\text { de mapas de un } \\
\text { videojuego) con sus } \\
\text { amigos virtuales y } \\
\text { usuarios Desconocidos: } \\
\text { "Es que yo quiero que se } \\
\text { pase bien, que la gente } \\
\text { que se lo pase bien" (Ja). }\end{array}$ & HOGAR \\
\hline $\begin{array}{l}\text { Creación de } \\
\text { Contenido } \\
\text { Digital }\end{array}$ & $\begin{array}{l}\text { Tiene su propio canal en YouTube, donde sube y } \\
\text { comparte vídeos. }\end{array}$ & $\begin{array}{l}\text { Quiere ser "creador de } \\
\text { videojuegos" (Ja), } \\
\text { porque le gusta y cree } \\
\text { que se le da bien. }\end{array}$ & HOGAR \\
\hline Seguridad & $\begin{array}{l}\text { La dimensión más débil: muestra desconfianza } \\
\text { de los adultos con los que comparte partidas. } \\
\text { Pero no sabe las contraseñas de sus dispositivos; } \\
\text { no conoce los requisitos de edad exigidos para } \\
\text { darse de alta en YouTube. Se comunica } \\
\text { frecuentemente con gente desconocida sin ser } \\
\text { consciente del posible peligro. }\end{array}$ & $\begin{array}{l}\text { Cree estar al tanto de los } \\
\text { riesgos de internet. } \\
\text { Afirma que no facilita } \\
\text { entrar en su cuenta a } \\
\text { otros usuarios y } \\
\text { manifiesta actitud } \\
\text { favorable hacia un uso } \\
\text { responsable y seguro de } \\
\text { los medios tecnológicos. }\end{array}$ & (AUSENCIA) \\
\hline $\begin{array}{l}\text { Resolución de } \\
\text { Problemas }\end{array}$ & $\begin{array}{l}\text { Resuelve incidencias en su entorno familiar con } \\
\text { los móviles o los videojuegos. También en el } \\
\text { aula con los ordenadores, cuando las páginas } \\
\text { web se quedan bloqueadas. }\end{array}$ & $\begin{array}{l}\text { Se considera hábil } \\
\text { resolviendo las } \\
\text { incidencias de los } \\
\text { móviles de sus } \\
\text { familiares, por ejemplo, } \\
\text { configurando cierta } \\
\text { canción como tono de } \\
\text { alarma en el teléfono de } \\
\text { su primo: "Sé yo más } \\
\text { que él" (Ja). }\end{array}$ & $\begin{array}{l}\text { HOGAR } \\
\text { ESCUELA }\end{array}$ \\
\hline
\end{tabular}

Aprendizajes ausentes en la Competencia Digital de preadolescentes: un estudio de casos pertenecientes a contextos socioculturales desfavorables. Fernando Fraga-Varela, Esther VilaCouñago y Eulogio Pernas-Morado. 
Las tablas mostradas permiten ver la conjugación entre el desarrollo de las dimensiones de la CD y los espacios donde aparecen las mismas. Los dos casos muestran carencias evidentes en varias dimensiones de la competencia, siendo claramente coincidentes en el apartado de Seguridad, pero extensible a Creación de Contenido Digital y Resolución de Problemas para Bieito. También vemos que en los dos casos, la dimensión que se trabaja explícitamente desde la escuela es la primera de Información y Alfabetización Informacional, claramente concurrente con lo que se indica en las entrevistas por parte del profesorado respecto a qué tipo de tareas se desarrollan en el aula con los portátiles de Abalar. Este trabajo de la escuela provoca que el resto de dimensiones, precisamente por la ausencia de trabajo, dependan del hogar para su desarrollo. Sólo en el caso de Jaime se da una aparente incoherencia al mostrar actividades de Resolución de Problemas en la escuela. Y decimos aparente porque es un aprendizaje que obedece claramente a lo que ha aprendido en el hogar.

\section{Conclusiones}

El análisis de los casos de Bieito y Jaime muestra la combinación de las circunstancias personales, sociales y escolares actuales que generan limitaciones para participar críticamente en el mundo digital en el que vivimos. El estudio localiza el origen de estas dificultades y cómo se afrontan gracias a la participación del profesorado del centro en la investigación, pero también a la observación de los casos en el trabajo con tecnología en el propio espacio escolar. Se evidencia que seguimos ante impedimentos en la escuela para avanzar en una alfabetización digital crítica (Pérez-Escoda et al., 2019) cuando existen políticas que se orientan desde hace tiempo precisamente en el abordaje de este tipo de aprendizajes. Se hacen visible las dudas sobre el significado de ser un nativo digital, ya que este hecho por sí mismo no ofrece ningún tipo de garantía (Sorrentino, 2018), persistiendo los indicios de falta de formación del alumnado ante este tipo de cuestiones (Lluna y Pedreira, 2017). La incorporación de la CD en su definición actual, siguiendo las propuestas de Ferrari (2013) y Vuorikari et al. (2016), precisa de una visión curricular renovada, ya que la actual estructura escolar en áreas de conocimiento tradicional y competencias de forma paralela, también la digital, trabajadas de forma independiente, no garantiza su articulación en verdaderos procesos de enseñanza y aprendizaje. Esta situación de dificultad no la encontramos en todas las competencias, ya que otras más próximas a las áreas impartidas tienen más facilidad de tener presencia a través de los contenidos curriculares. Se presenta un escenario con buenas intenciones, pero en el que falta un trabajo de mayor definición.

Así lo evidencian los casos. Bieito posee un desarrollo bajo de la CD. Una situación de la que la familia no es consciente, pero sí a ojos de la institución escolar. Porque no se trata exclusivamente de lo digital: las dificultades son generalizadas en el conjunto de los aprendizajes que un niño de su edad debería haber desplegado. De todo el abanico de las dimensiones que caracterizan a la $\mathrm{CD}$, sólo vemos desarrollos muy parciales en dos dimensiones de cinco (información y alfabetización informacional; comunicación y colaboración), con tres ausencias muy reveladoras. La situación mostrada también evidencia que la escolarización de Bieito no garantiza una compensación en estas limitaciones, debido a las diferentes demandas a las que se enfrenta la escuela. En cuanto a Jaime, podríamos representar su CD haciendo alusión a un terreno más fértil -su habilidad para el uso de las tecnologías ha quedado puesta de manifiesto de manera notoria-, pero fragmentado, en el que algunas dimensiones sí se han cosechado -aunque

Aprendizajes ausentes en la Competencia Digital de preadolescentes: un estudio de casos pertenecientes a contextos socioculturales desfavorables. Fernando Fraga-Varela, Esther VilaCouñago y Eulogio Pernas-Morado. 
no en una posición coherente de familia y escuela- y en otras apenas se ha intervenido: la seguridad y la resolución de problemas son las menos desarrolladas. Los pocos nexos que encontramos se concentran en el área de "Información y Alfabetización Informacional", promovida desde los contextos escolar y familiar, y en el área de "Comunicación y Colaboración", la cual se desenvuelve a nivel familiar y con desconocidos a través de la virtualidad en el mundo de los videojuegos. Para Jaime sería preciso repensar qué se entiende por grupo de iguales, porque en su caso, si bien no tiene referentes en este sentido en su día a día familiar y social (su padre es la figura clave gestionando su ocio y su tiempo personal), dichos iguales parecen haberse trasladado hacia lo virtual. Estamos ante un desarrollo muy básico y parcial de su red personal de aprendizaje (PLN), sustentado únicamente en conocidos de su grupo de amigos virtuales (y otros usuarios) con los que coincide en sus juegos digitales, pero que no llegan a conformar un grupo de iguales claramente referenciable.

Los casos trabajados nos confirman las dificultades en este tipo de aprendizajes (AlonsoFerreiro y Gewerc, 2018), con el agravante de encontrarse escolarizados en centros de saturación tecnológica con equipamiento 1 a 1 . Una situación ya recogida en anteriores trabajos en espacios de estas características (Area et al., 2014). Sin embargo, lo novedoso a la luz de los casos es poner en valor el papel que se requiere por parte de las familias para que se puedan llevar a mejor puerto las propuestas centradas en la CD. Aparece así el capital cultural como elemento básico de comprensión en la apropiación de las tecnologías digitales disponibles y marco estructurante de las competencias desarrolladas con estas tecnologías desde el hogar. Los niños y las niñas se inician ya desde muy pequeños a través de los tiempos de ocio en el desarrollo de la $\mathrm{CD}$, por imitación del comportamiento de otros miembros de la familia, donde se ve la influencia de los modelos de consumos privilegiados en el hogar. Y aun así, si bien realizan un uso sistemático de tecnologías digitales en el ocio, desarrollan habilidades, pero no necesariamente competencias.

El estudio de los casos de Bieito y Jaime, poniendo el foco en la CD, también nos muestra la falta de sinergia entre escuela y familia: las actividades relacionadas con esta competencia discurren por caminos paralelos, cuando no divergentes. Este es un aspecto que la administración educativa debe acometer con urgencia en beneficio del alumnado. $\mathrm{Y}$ es que parece imposible un desarrollo integral de la CD sin tener en cuenta el papel de las familias y sin que se haga un esfuerzo para la colaboración conjunta, por ejemplo, en temas tan delicados como la seguridad ante los riesgos de las redes o la protección de la identidad de los menores. Encontrar, desarrollar y favorecer el empleo de espacios (tal vez también virtuales) de colaboración conjunta y herramientas para una efectiva comunicación entre las familias y los centros escolares, puede ser una buena solución; en especial debido a la progresiva implantación en las escuelas de herramientas como aulas virtuales para la enseñanza y el aprendizaje, los libros digitales o las apps.

Los casos contribuyen a la hipótesis general del Proyecto CDEPI: consideramos que se están generando importantes desequilibrios en la adquisición de la CD en función de los diferentes entornos familiares y sociales del alumnado. Si bien la escuela podría ser un lugar privilegiado y fundamental para el desarrollo de la $\mathrm{CD}$, tiene dificultades para su enseñanza. Una situación familiar de desestructuración puede suponer un gran impacto en la adquisición de las competencias, un hecho ante el cual la escuela se encontraría con serios problemas, con los tiempos y espacios actuales, para abordar los obstáculos y compensarlos. Ante esta situación, las brechas no se reparan, incluso se acrecientan en un

Aprendizajes ausentes en la Competencia Digital de preadolescentes: un estudio de casos pertenecientes a contextos socioculturales desfavorables. Fernando Fraga-Varela, Esther VilaCouñago y Eulogio Pernas-Morado. 
escenario como el actual, con una complejidad creciente y en el que no se muestran otros tipos de mecanismos sociales de apoyo que puedan compensar ese desequilibrio.

Presentación del artículo: 15 de octubre de 2019

Fecha de aprobación: 8 de noviembre de 2019

Fecha de publicación: 30 de noviembre de 2019

Fraga-Varela, F., Vila-Couñago, E. y Pernas-Morado, E. (2019). Aprendizajes ausentes en la Competencia Digital de preadolescentes: un estudio de casos pertenecientes a contextos socioculturales desfavorables. RED. Revista Educación a Distancia, 61. DOI: http://dx.doi.org/10.6018/red/61/04

\section{Financiación}

El Proyecto "Competencia digital en estudiantes de educación obligatoria. Entornos sociofamiliares, procesos de apropiación y propuestas de e-inclusión" (CDEPI) es una investigación financiada por el Ministerio de Economía y Competitividad (Ref.: EDU2015-67975-C3-1-P) y por el Fondo Europeo de Desarrollo Regional (FEDER).

\section{Referencias}

Alonso-Ferreiro, A. y Gewerc, A. (2018). Alfabetización mediática en la escuela primaria. Estudio de caso en Galicia. Revista complutense de educación, 29(2), 407422. DOI https://doi.org/10.5209/RCED.52698

Area, M. y Pessoa, T. (2012). De lo sólido a lo líquido: las nuevas alfabetizaciones ante los cambios culturales de la Web 2.0. Revista Comunicar, 19(38), 13-20. DOI https://doi.org/10.3916/C38-2012-02-01

Area Moreira, M. et al. (2014). Las políticas educativas TIC en España después del Programa Escuela 2.0: las tendencias que emergen. RELATEC: Revista Latinoamericana de Tecnología Educativa, 13(2), 11-33. DOI https://doi.org/ 10.17398/1695-288X.13.2.11

Bennett, S. J., Maton, K. A. y Kervin, L. K. (2008). The 'digital natives' debate: a critical review of the evidence. British Journal of Educational Technology, 39(5), 775-786. DOI https://doi.org/10.5209/RCED.52698

Bawden, D. (2008). Origins and concepts of digital literacy. Digital literacies: Concepts, policies and practices, 30, 17-32.

Buckingham, D. (2007). Digital Media Literacies: rethinking media education in the age of the Internet. Research in Comparative and International Education, 2(1), 43-55. DOI https://doi.org/10.2304/rcie.2007.2.1.43

Cobo, C. (2019). Acepto las condiciones: usos y abusos de las tecnologías digitales. Madrid: Fundación Santillana.

Coller, X. (2005). Estudio de casos (2a ed.). Madrid: CIS.

Aprendizajes ausentes en la Competencia Digital de preadolescentes: un estudio de casos pertenecientes a contextos socioculturales desfavorables. Fernando Fraga-Varela, Esther VilaCouñago y Eulogio Pernas-Morado.

Página 16 de 18 
Couros, A. (2010). Developing personal learning networks for open and social learning. En Veletsianos, G. (Ed.), Emerging technologies in distance education (pp. 109128). Edmonton, Alberta: Athabasca University Press. Disponible en http://www.aupress.ca/books/120177/ebook/06_Veletsianos_2010Emerging_Technologies_in_Distance_Education.pdf

Creswell, J. W. y Plano, V. L. (2018). Designing and conducting mixed methods research $\left(3^{a}\right.$ ed.). Thousand Oaks, CA: Sage.

Díaz-Vicario, A., Mercader, C. y Gairín, J. (2019). Uso problemático de las TIC en adolescentes. Revista Electrónica de Investigación Educativa, 21(e07), 1-11. DOI https://doi.org/10.24320/redie.2019.21.e07.1882

Ferrari, A. (2013). DIGCOMP: A Framework for Developing and Understanding Digital Competence in Europe. Sevilla: Joint Research Centre, Institute for Prospective Technological Studies. DOI https://doi.org/10.2788/52966

Gallardo-Echenique, E. E. (2019). Brechas y asimetrías que emergen en la era digital, ¿nuevas formas de exclusión? Revista Electrónica de Investigación Educativa, 21(3), 1-3. Recuperado de http://redie.uabc.mx/redie/article/view/2909

Glaser, B. G. y Strauss, A. L. (1967). The Discovery of grounded theory: strategies for qualitative research. New York: Aldine Publishing. DOI https://doi.org/10.1097/00006199-196807000-00014

Helsper, E. J. y Eynon, R. (2009). Digital natives: where is the evidence? British educational research journal, 36(3), 503-520. DOI https://dx.doi.org/10.1080/ 01411920902989227

Jenkins, H. (2009). Confronting the challenges of participatory culture: media education for the 21st century. Cambridge, MA: The MIT Press. DOI https://doi.org/10.7551/mitpress/8435.001.0001

Knobel, M. y Lankshear, C. (2008). Digital literacy and participation in online social networking spaces. En C. Lankshear y M. Knobel (Eds.), Digital literacies: Concepts, policies and practices (pp. 249-278). New York: Peter Lang.

Ley Orgánica 2/2006, de 3 de mayo, de Educación. BOE, 4 de mayo, núm. 106, pp. 17158-17207. Disponible en https://www.boe.es/buscar/act.php?id=BOE-A-20067899

Ley Orgánica 8/2013, de 9 de diciembre, para la Mejora de la Calidad Educativa. BOE, 10 de diciembre, núm. 295, pp. 97858-97921. Disponible en https://www.boe.es/buscar/act.php?id=BOE-A-2013-12886

Lluna, S. y Pedreira, J. (2017). Los nativos digitales no existen. Cómo educar a tus hijos para un mundo digital. Barcelona: Deusto.

Linne, J. W. (2018). Nomadización, ciudadanía digital y autonomía. Tendencias juveniles a principios del siglo XXI. Chasqui. Revista Latinoamericana de Comunicación, (137), 37-52. Recuperado de https://revistachasqui.org/index.php/chasqui/article/ download/3381/3010

McGrew, S., Breakstone, J., Ortega, T. Smith, M. y Wineburg, S. (2018). Can students evaluate online sources? Learning from assessments of civic online reasoning.

Aprendizajes ausentes en la Competencia Digital de preadolescentes: un estudio de casos pertenecientes a contextos socioculturales desfavorables. Fernando Fraga-Varela, Esther VilaCouñago y Eulogio Pernas-Morado. 
Theory \& Research in Social Education, 46(2), 165-193. DOI https://doi.org/10.1080/00933104.2017.1416320

Muñoz Justicia, J. y Sahagún Padilla, M. (2017). Hacer análisis cualitativo con Atlas.ti 7. Manual de uso. Recuperado de http://manualatlas.psicologiasocial.eu/atlasti7.pdf

Oddone, K., Hughes, H. y Lupton, M. (2019). Teachers as Connected Professionals: A Model to Support Professional Learning Through Personal Learning Networks. International Review of Research in Open and Distributed Learning, 20(3), 103120. DOI https://doi.org/10.19173/irrodl.v20i4.4082

ORGANISATION FOR ECONOMIC COOPERATION AND DEVELOPMENT (2001). Understanding the Digital Divide. OECD Digital Economy Papers, no 49. Recuperado de http://www.oecd.org/dataoecd/38/57/1888451.pdf

Pérez-Escoda, A., García-Ruiz, R. y Aguaded, I. (2019). Dimensiones de la alfabetización digital a partir de cinco modelos de desarrollo. Cultura y Educación, 31(2), 232266. DOI https://doi.org/10.1080/11356405.2019.1603274

Prensky, M. (2001). Digital Natives, Digital Immigrants. On the Horizon, 9(5), 1-6. DOI https://doi.org/10.1108/10748120110424816

Sorrentino, P. (2018). The mystery of the digital natives' existence: Questioning the validity of the Prenskian metaphor. First Monday, 23(10). DOI https://doi.org/ 10.5210/fm.v23i10.9434

Stake, R. E. (2010). Investigación con estudio de casos ( $5^{\mathrm{a}}$ ed.). Madrid: Morata.

Tobin, D. (1998). Building Your Personal Learning Network. Corporate Learning Strategie. Recuperado de http://tobincls.com/wp-content/uploads/2017/01/ building-a-personal-learning-network.docx

Tójar, J. C. (2006). Investigación cualitativa. Comprender y actuar. Madrid: La Muralla.

Van Deursen, A. J. y Van Dijk, J. A. (2014). The digital divide shifts to differences in usage. New media \& society, 16(3), 507-526. DOI https://doi.org/10.1177/ 1461444813487959

Velásquez Benavides, A., Rodríguez Hidalgo, C. y Suing, A. (2018). Jóvenes y cultura digital ¡Siguen los cambios y sin miedos! Chasqui. Revista Latinoamericana de Comunicación, (137), 33-37. Recuperado de https://revistachasqui.org/index.php/ chasqui/article/view/3667

Vuorikari, R., Punie, Y., Carretero, S. y Van den Brande, G. (2016). DigComp 2.0: The Digital Competence Framework for Citizens. Update Phase 1: The Conceptual Reference Model. Luxembourg Publication Office of the European Union. DOI https://doi.org/10.2791/11517

Warlick, D. (2009). Grow Your Personal Learning Network: New Technologies Can Keep You Connected and Help You Manage Information Overload. Learning \& Leading with Technology, 36(6), 12-16.

Yin, R. K. (2009). Case study research. Design and methods ( $4^{\mathrm{a}}$ ed.). Londres: Sage.

Aprendizajes ausentes en la Competencia Digital de preadolescentes: un estudio de casos pertenecientes a contextos socioculturales desfavorables. Fernando Fraga-Varela, Esther VilaCouñago y Eulogio Pernas-Morado.

Página 18 de 18 